\title{
Reliability of Early Ambulation after Intradural Spine Sur- gery : Risk Factors and a Preventive Method for Cerebro- spinal Fluid Leak Related Complications
}

\author{
Subum Lee, ${ }^{1}$ Dae-Chul Cho, Kyoung-Tae Kim, ${ }^{1}$ Young-Seok Lee, ${ }^{2}$ Seung Chul Rhim, ${ }^{3}$ Jin Hoon Park ${ }^{3}$ \\ Department of Neurosurgery, Kyungpook National University Hospital, School of Medicine, Kyungpook National University, Daegu, Korea \\ Department of Neurosurgery, Kyungpook National University Chilgok Hospital, School of Medicine, Kyungpook National University, Daegu, \\ Korea \\ Department of Neurosurgery, ${ }^{3}$ Asan Medical Center, University of Ulsan College of Medicine, Seoul, Korea
}

Objective : Cerebrospinal fluid leakage related complications (CLC) occasionally occur after intradural spinal surgery. We sought to investigate the effectiveness of early ambulation after intradural spinal surgery and analyze the risk factors for CLC.

Methods : For this retrospective cohort study, we enrolled 314 patients who underwent intradural spinal surgery at a single institution. The early group contained 79 patients who started ambulation after 1 day of bedrest without position restrictions, while the late group consisted of 235 patients who started ambulation after at least 3 days of bed rest and were limited to the prone position after surgery. In the early group, Prolene $6-0$ was used as the dura suture material, while black silk 5-0 was used as the dura suture material in the late group.

Results : The overall incidence rate of CLC was $10.8 \%$. Significant differences between the early and late groups were identified in the rate of CLC ( $2.5 \%$ vs. $13.6 \%)$, surgical repair required (1.3\% vs. $7.7 \%)$, and length of hospital stay ( 2.99 vs. 9.29 days) $(p<0.05)$. Logistic regression analysis revealed that CLC was associated with practices specific to the late group $(p=0.011)$ and the revision surgery $(p=0.022)$.

Conclusion : Using Prolene 6-0 as a dura suture material for intradural spinal surgery resulted in lower CLC rates compared to black silk 5-0 sutures despite a shorter bed rest period. Our findings revealed that suture - needle ratio related to dura defect was the most critical factor for CLC. One-day ambulation after primary dura closure using Prolene 6-0 sutures appears to be a costeffective and safe strategy for intradural spinal surgery.

Key Words : Cerebrospinal fluid leak · Complications · Dural repair · Postoperative care · Spinal cord neoplasms.

\section{INTRODUCTION}

Cerebrospinal fluid (CSF) leakage related complications
(CLC) occur after intradural spinal surgery at an incidence rate that ranges from $1.5 \%$ to $13 \%{ }^{7,14,15,23)}$. Postoperative CSF leakage often requires secondary interventions, and CLC after

- Received : December 16, 2020 •Revised : January 29, 2021 •Accepted : February 26, 2021

- Address for reprints : Jin Hoon Park

Department of Neurological Surgery, Asan Medical Center, University of Ulsan College of Medicine, 88 Olympic-ro 43-gil, Songpa-gu, Seoul 05505, Korea Tel : +82-2-3010-3550, Fax : +82-2-476-6738, E-mail : jhpark@amc.seoul.kr, ORCID : https://orcid.org/0000-0002-0903-3146 
spinal surgery constitute a major source of patient morbidity and economic burden ${ }^{27)}$. In some cases, wound healing is delayed due to pseudomeningocele or durocutaneous fistulas, which may require re-operation. CLC may also extend the duration of the inpatient postoperative care period. Prolonged bed rest decreases the CSF pressure at the site of the durotomy, thereby reducing the flow of CSF through the dural defect and potentially reducing these complications ${ }^{2,3,5}$. However, there is insufficient evidence from which to form a consensus among spinal surgeons. Moreover, complications of bed rest in the prone position, such as thromboembolism, should not be overlooked $^{4,22)}$.

Although there are a number of risk factors that may contribute to the occurrence of CLC, they are not well defined ${ }^{2}$. A watertight closure and proper suture material are critical factors for an effective intraoperative management strategy ${ }^{6}$. In one large scale study, a 98.2\% success rate was obtained using 5-0 silk sutures for a dura tear ${ }^{16}$. In contrast, $6-0$ Prolene demonstrated the best results for watertight closure in recent experimental research on the hydrostatic strength of dural repairs using various suture sizes ${ }^{1,4)}$.

So far, there have been no direct comparative clinical studies of postoperative bed rest periods and respective suture materials in a single-center setting. In the present study, we evaluated the efficacy of postoperative 1-day ambulation compared to a more extended bed rest period as a routine postoperative care protocol following intradural spinal surgery. We furthermore investigated the risk factors for the occurrence of CLC.

\section{MATERIALS AND METHODS}

This study received approval from the Institutional Review Board of Asan Medical Center (IRB approval No. AMC IRB 2020-0711).

\section{Study design}

We conducted a retrospective cohort study to evaluate the effects of dura suture material and postoperative bed rest after intradural spinal surgery. The patients were divided into an early ambulation (early) group, comprising patients who start ambulation on the first day after surgery, and a late ambulation (late) group, comprising patients who start ambulation on the third day after surgery or later, depending on wound status. In the early group, polypropylene 6-0 Prolene sutures

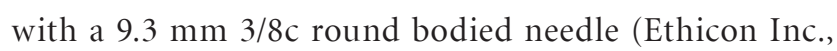
Somerville, NJ, USA) were used as the dural suture material. Patients who had surgery between March 2018 and December 2019 were included in this group. In the late group, braided non-absorbable $5-0$ black silk sutures with a $10 \mathrm{~mm} \mathrm{3/8c}$ round bodied needle (Ailee, Busan, Korea) were used as the dural suture material. Patients who had surgery between January 2012 and December 2018 were included in this group. Clinical characteristics, pathology, surgical outcomes, CLC, and surgical repair rate were compared between the groups.

\section{Study participants and data collection}

We reviewed all patients who underwent intradural spinal surgery in Asan Medical Center between January 2012 and December 2019. The inclusion criteria were as follows : 1) patients with intradural tumors or vascular malformations; 2) patients who underwent posterior midline approach and durotomy after laminectomy or laminotomy; 3) patients in whom primary dura closure was performed; and 4) those with a follow-up period $\geq 6$ months. Patients who had surgery due to trauma or infectious causes, extradural lesions, or who had undergone additional anterior surgical procedures, duroplasty, or incomplete dura closure, as well as patients who had simple surgical site infections without CSF leaks were excluded. Operations on patients included in the early group were performed by a junior surgeon (name-blinded) and operations on patients in the late group were performed by a senior surgeon (name-blinded). Patients attended a postoperative outpatient clinic at 1 month (or earlier if necessary) and 6 months post-surgery for assessment of clinical symptoms and wound status follow-up.

Clinical data were obtained from our institutional electronic medical records. Patient age, sex, body mass index, smoking, diabetes mellitus, antiplatelet or anticoagulation agent status, pathologic diagnosis, location, revisional skin incision, and durotomy associated with a previous scar or first surgery were recorded. To evaluate the surgical outcomes and complications after surgery, we also recorded data on the performance and level of laminectomy or laminotomy, postoperative hospital days, bedrest days, and McCormick grade. 

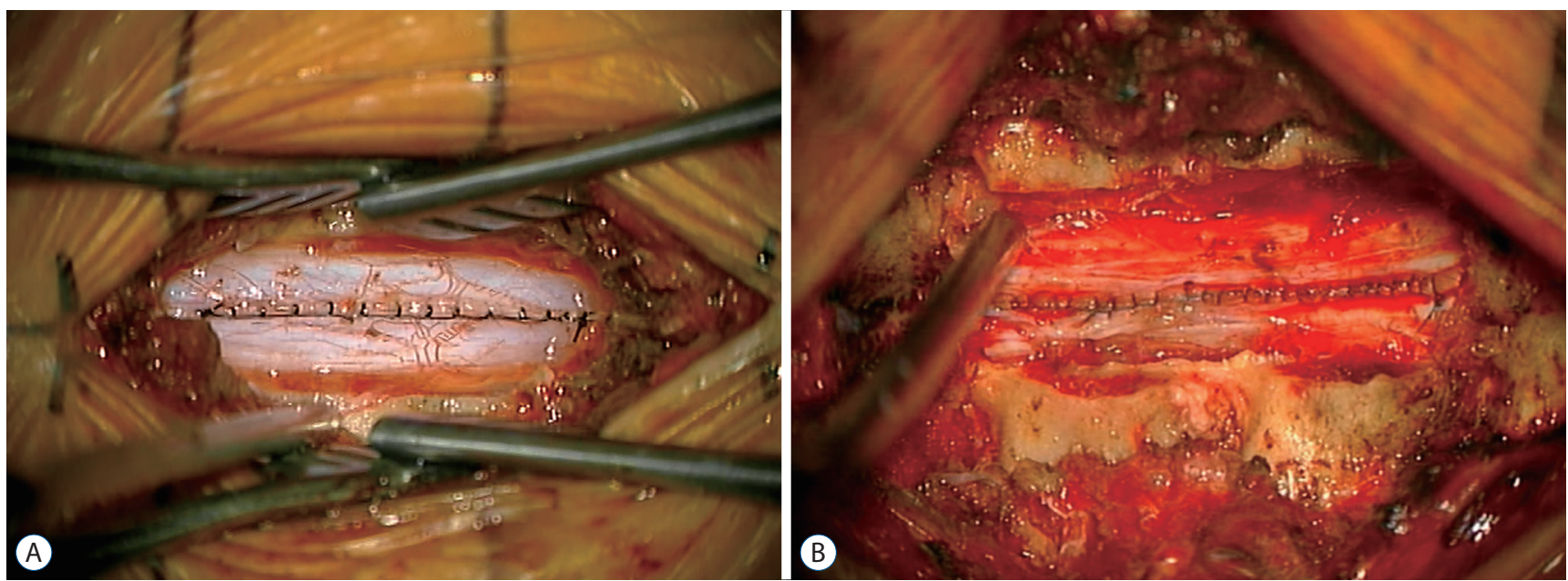

Fig. 1. Intraoperative microscopic image of dura closure performed using running locked suture technique. Prolene 6-0 suture was used for the early group patients (A); black silk 5-0 suture was used for the late group patients (B).

Table 1. Patient demographics and clinical characteristics

\begin{tabular}{|c|c|c|c|c|}
\hline Variable & Early group $(n=79)$ & Late group $(n=235)$ & Total & $p$-value \\
\hline Age (years)* & $55.62 \pm 16.13$ & $50.70 \pm 14.71$ & & 0.013 \\
\hline Sex, male : female & $36: 43$ & $95: 140$ & & 0.422 \\
\hline Body mass index $\left(\mathrm{kg} / \mathrm{m}^{2}\right)$ & $24.67 \pm 3.57$ & $24.34 \pm 3.95$ & & 0.509 \\
\hline Smoking & $11(13.9)$ & $55(23.4)$ & & 0.074 \\
\hline Diabetes mellitus & $7(8.9)$ & $30(12.8)$ & & 0.352 \\
\hline Antiplatelet or anticoagulant & $2(2.5)$ & $10(4.3)$ & & 0.737 \\
\hline Revision & $5(6.3)$ & $9(3.8)$ & & 0.352 \\
\hline Intramedullary/extramedullary* & 18/61 (22.8) & 29/206 (12.3) & & 0.024 \\
\hline Location & & & & 0.964 \\
\hline Cervical & $26(32.9)$ & $64(27.2)$ & $90(28.7)$ & \\
\hline Cervico-thoracic junction & $3(3.8)$ & $13(5.5)$ & $16(5.1)$ & \\
\hline Thoracic & $23(29.1)$ & $71(30.2)$ & $94(29.9)$ & \\
\hline Thoraco-lumbar junction & $5(6.3)$ & $16(6.8)$ & $21(6.7)$ & \\
\hline Lumbar & $21(26.6)$ & $67(28.5)$ & $88(28.0)$ & \\
\hline Sacral & $1(1.3)$ & $4(1.7)$ & $5(1.6)$ & \\
\hline Pathology & & & & 0.552 \\
\hline Ependymoma & $4(5.1)$ & $21(8.9)$ & $25(8.0)$ & \\
\hline Astrocytoma & $2(2.5)$ & $2(0.9)$ & $4(1.3)$ & \\
\hline Hemangioblastoma & $4(5.1)$ & $12(5.1)$ & $16(5.1)$ & \\
\hline Meningioma & $14(17.7)$ & $43(18.3)$ & $57(18.2)$ & \\
\hline Neurofibroma & $1(1.3)$ & $4(1.7)$ & $5(1.6)$ & \\
\hline Schwannoma & $42(53.2)$ & $132(56.2)$ & $174(55.4)$ & \\
\hline Vascular malformation & $5(6.3)$ & $6(2.6)$ & $11(3.5)$ & \\
\hline Myxopapillary ependymoma & $1(1.3)$ & $5(2.1)$ & $6(1.9)$ & \\
\hline Others & $6(7.6)$ & $10(4.3)$ & $16(5.1)$ & \\
\hline
\end{tabular}

Values are presented as mean \pm standard deviation or number (\%) unless otherwise indicated. *Statistically significant difference $(p<0.05)$ 


\section{Surgical technique and postoperative manage- ment}

Dura closures were all performed using a running locked suture technique (Fig. 1). The throws were approximately 1 to $2 \mathrm{~mm}$ apart and these were tied with a surgeon's knot at each end under microscopic magnification using microsurgical instruments (Castroviejo needle holders, Gerald tissue forceps). Following completion of the dura closure, a Valsalva maneuver was used to check for CSF leakage, and the fibrin sealant patch (TachoSil, Baxter, CA, USA) was applied. There was an intentional effort to perform laminotomy to the maximum

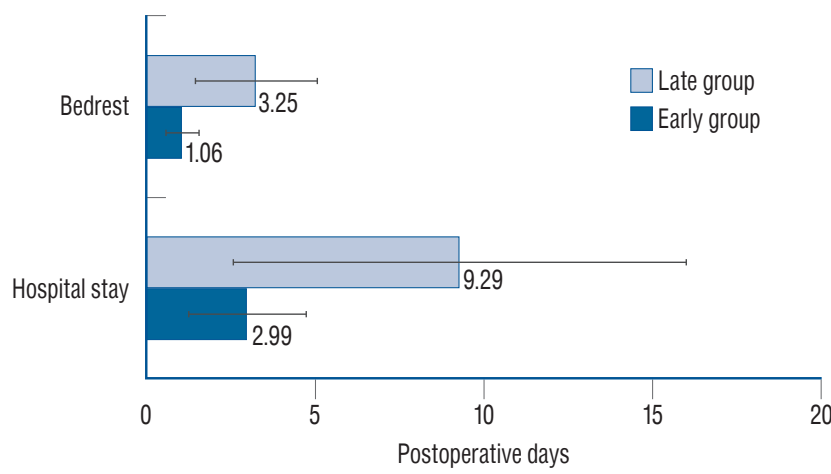

Fig. 2. Early group patients were discharged significantly faster (postoperative 2.99 days vs. 9.29 days, $p<0.001$ ), they started ambulation earlier after sit up (postoperative 1.06 days vs. 3.25 days, $p<0.001$ ). Despite the shorter duration of the early group's hospital stay, the overall $\mathrm{CLC}$ rate was low. CLC : cerebrospinal fluid leakage related complications. possible extent on patients in the early group. Laminoplasties were performed using Lorenz Plating System Neuro Implants (Biomet, Jacksonville, FL, USA) or Centerpiece Hinge plates (Medtronic, Memphis, TN, USA). No patient in this study received the placement of a subfascial wound drain. The muscle fascia was closed as tightly as possible using 1-0 Vicryl sutures (Ethicon Inc.).

Early group patients routinely started walking on the first day after surgery, and tolerable ambulation was maintained when symptoms such as hypotensive headache occurred. Late group patients routinely stayed on bedrest in the prone position for 3 days, with daily monitoring of the condition of the wound. In cases where fluid collection or wound bulging was observed, the bed rest period was prolonged. In the late group only, a lumbar drain was considered when CSF leakage occurred or when a definite pseudomeningocele was present.

\section{Statistical analysis}

Variables between the early and late groups were compared using an independent t-test, Mann-Whitney $U$ test, and chisquared test. To adjust for confounding variables, a multivariate logistic regression analysis was performed on the risk factors associated with CLC that were identified as significant in a simple logistic regression analysis. The odds ratios (ORs) of the risk factors were obtained using the chi-squared test. All data management and statistical analyses were performed us-

Table 2. Surgical outcomes and complications

\begin{tabular}{|c|c|c|c|}
\hline Variable & Early group $(n=79)$ & Late group $(n=235)$ & $p$-value \\
\hline Surgical level & $2.05 \pm 1.11$ & $2.01 \pm 0.98$ & 0.750 \\
\hline Laminectomy/laminotomy* & $9(11.4) / 70(88.6)$ & $115(48.9) / 120(51.1)$ & $<0.001$ \\
\hline In hospital post op care (POD)* & $2.99 \pm 1.74$ & $9.29 \pm 6.73$ & $<0.001$ \\
\hline Post op sit up (POD)* & $1.06 \pm 0.49$ & $3.25 \pm 1.81$ & $<0.001$ \\
\hline Pre-McCormick grade* & $2.11 \pm 1.00$ & $1.46 \pm 0.71$ & $<0.001$ \\
\hline Post McCormick grade & $1.62 \pm 0.79$ & $1.78 \pm 0.74$ & 0.094 \\
\hline PTE \& DVT & $0(0.0)$ & $2(0.9)$ & 1.000 \\
\hline lleus* & $1(1.3)$ & $20(8.6)$ & 0.025 \\
\hline Hypotensive headache & $6(7.6)$ & $21(8.9)$ & 0.713 \\
\hline CSF leak related complication* (pseudomeningocele, fistula) & $2(2.5)$ & $32(13.6)$ & 0.006 \\
\hline Lumbar drain insertion & $0(0.0)$ & $10(4.3)$ & 0.062 \\
\hline Additional revision surgery* (dura repair and wound revision) & $1(1.3)$ & $18(7.7)$ & 0.039 \\
\hline
\end{tabular}

Values are presented as mean \pm standard deviation or number (\%) unless otherwise indicated. *Statistically significant difference ( $p<0.05)$. POD : postoperative day, PTE : pulmonary thromboembolism, DVT : deep vein thrombosis, CSF : cerebrospinal fluid 
ing SPSS software (version 20.0; IBM Corporation, Armonk, NY, USA). A $p$-value $<0.05$ was considered significant.

\section{RESULTS}

\section{Patient demographics and clinical characteristics}

There were no differences in sex, smoking, diabetes mellitus, use of anticoagulants or antiplatelet agents, revision surgeries (when approaching through the previous wound), or distribution of surgical location. The average age was significantly higher in the early group (55.62 vs. 50.70 years, $p=0.013)$, as was the rate of intramedullary lesions $(22.8 \%$ vs. $12.3 \%, p=0.024)$. Intradural lesions consisted of tumors and vascular malformations (cavernoma, arteriovenous fistula), and did not differ significantly between the two groups. Schwannomas (55.4\%) were the most common, followed by meningiomas and ependymomas (Table 1).

\section{Surgical outcomes and complication}

The range of surgical levels did not differ between the two groups, and laminoplastic laminotomy was performed at a higher rate in the early group patients than in the late group patients $(88.6 \%$ vs. $51.1 \%, p<0.001)$. Patients in the early group were discharged significantly faster (postoperative 2.99 days vs. 9.29 days, $p<0.001)$. They also achieved ambulation earlier after sitting up (postoperative 1.06 days vs. 3.25 days, $p<0.001$ ) (Fig. 2).

Since most of the diseases included in this study were spinal cord tumors, we utilized the McCormick grade for the comparison of neurologic outcomes. The early group demonstrated a higher grade prior to surgery (2.11 vs. $1.46, p<0.001$ ), but there was no significant difference between the two groups after surgery (1.62 vs. $1.78, p=0.094$ ).

Ileus is a complication that may occur in relation to bed rest after surgery. In the early group, the rate of ileus was significantly lower compared to the late group $(1.3 \%$ vs. $8.6 \%$, $p=0.025$ ). The rate of fatal complications such as pulmonary thromboembolism and deep vein thrombosis did not differ significantly between groups. There were two cases in the late group and no cases in the early group. There was also no difference in the frequency of hypotensive headache, one of the symptoms of CSF leakage (7.6\% vs. $8.9 \%, p=0.713)$. CLC including symptomatic pseudomeningocele, durocutaneous fis- tula, and wound dehiscence with oozing were significantly reduced in the early group $(2.5 \%$ vs. $13.6 \%, p=0.006)$. In the late group, 10 patients had a lumbar drain after surgery, while

Table 3. Univariate logistic regression analysis of risk factors for CLC

\begin{tabular}{|c|c|c|c|}
\hline & OR & $95 \% \mathrm{Cl}$ & $p$-value \\
\hline Age & 1.004 & $0.980-1.028$ & 0.745 \\
\hline Sex, male & 1.663 & $0.814-3.397$ & 0.163 \\
\hline 1st assistant, fellows & & & 0.052 \\
\hline$A$ & 1.000 & & \\
\hline B & 2.042 & $0.771-5.408$ & 0.151 \\
\hline C & 0.868 & $0.239-3.152$ & 0.829 \\
\hline $\mathrm{D}$ & 0.000 & $0.000-0.000$ & 0.999 \\
\hline$E$ & 0.556 & $0.171-1.800$ & 0.327 \\
\hline $\mathrm{F}$ & 0.120 & $0.014-0.991$ & 0.049 \\
\hline G & 0.000 & $0.000-0.000$ & 0.997 \\
\hline $\mathrm{H}$ & 0.260 & $0.053-1.286$ & 0.099 \\
\hline Group, late* & 6.069 & $1.420-25.936$ & 0.015 \\
\hline Post op sit up (POD)* & 1.570 & $1.300-1.897$ & $<0.001$ \\
\hline Revision case, yes* & 3.600 & $1.064-12.186$ & 0.039 \\
\hline Intramedullary, yes & 1.249 & $0.487-3.204$ & 0.643 \\
\hline Surgical level & & & 0.980 \\
\hline $1(n=105)$ & 1.000 & & \\
\hline $2(n=140)$ & 1.181 & $0.528-2.641$ & 0.685 \\
\hline $3(n=40)$ & 0.693 & $0.183-2.625$ & 0.589 \\
\hline $4(n=20)$ & 0.949 & $0.194-4.650$ & 0.949 \\
\hline $5(n=8)$ & 1.221 & $0.137-10.869$ & 0.858 \\
\hline $7(n=1)$ & 0.000 & $0.000-0.000$ & 1.000 \\
\hline Location & & & 0.223 \\
\hline Cervical & 1.000 & & \\
\hline Cervico-thoracic junction & 4.091 & $1.153-14.446$ & 0.029 \\
\hline Thoracic & 0.837 & $0.308-2.275$ & 0.728 \\
\hline Thoraco-lumbar junction & 0.947 & $0.189-4.747$ & 0.948 \\
\hline Lumbar & 1.025 & $0.387-2.717$ & 0.960 \\
\hline Sacral & 2.250 & $0.226-22.374$ & 0.489 \\
\hline Laminectomy, yes & 1.847 & $0.903-3.777$ & 0.093 \\
\hline BMI & 1.042 & $0.951-1.142$ & 0.377 \\
\hline Diabetes mellitus, yes & 2.160 & $0.867-5.386$ & 0.098 \\
\hline Smoking, yes & 1.957 & $0.900-4.254$ & 0.090 \\
\hline $\begin{array}{l}\text { Antiplatelet or } \\
\text { anticoagulation agent, yes }\end{array}$ & 2.914 & 0.749-11.336 & 0.123 \\
\hline
\end{tabular}

*Statistically significant difference $(p<0.05)$. CLC : cerebrospinal fluid leakage related complications, $\mathrm{OR}$ : odds ratio, $\mathrm{Cl}$ : confidence interval, POD : postoperative day, BMI : body mass index 
Table 4. Multivariate logistic regression analysis of risk factors for $\mathrm{CLC}$

\begin{tabular}{|c|c|c|c|}
\hline & \multicolumn{2}{|c|}{ Multivariate analysis } & \multirow{2}{*}{$p$-value } \\
\hline & OR & $95 \% \mathrm{Cl}$ & \\
\hline Group, late* & 6.703 & $1.540-29.176$ & 0.011 \\
\hline Revision case, yes* & 4.537 & $1.246-16.523$ & 0.022 \\
\hline
\end{tabular}

*Statistically significant difference $(p<0.05)$; factors with $p<0.100$ in univariate logistic regression analysis were entered and backward elimination (Likelihood ratio) method was used. CLC : cerebrospinal fluid leakage related complications, $\mathrm{OR}$ : odds ratio, $\mathrm{Cl}$ : confidence interval

there were no cases in the early group that required a lumbar drain. Despite meticulous conservative treatment and close observation, one patient $(1.3 \%)$ in the early group underwent additional repair surgery due to worsening progression, compared to 18 patients $(7.7 \%)$ in the late group, a difference that was statistically significant ( $p=0.039$ ) (Table 2 ).

\section{Risk factors of CLC}

In simple logistic regression analysis, an increased incidence of postoperative CLC was significantly associated with the late group ( $p=0.015)$, as well as day of postoperative sit-up $(p<0.001)$, and re-operation $(p=0.039)$ (Table 3$)$. Assignment to the late group $(p=0.011)$ and revision surgery $(p=0.022)$ remained significant risk factors for postoperative CLC following multivariate analysis (Table 4).

\section{DISCUSSION}

This study sought to investigate the efficacy of routine 1-day ambulation after intradural spinal surgery. We compared two groups that differed in the suture material used for dural closure and in strategies for postoperative care, according to the policies of each surgeon.

Patient positioning after a durotomy remains controversial. Conventional wisdom suggests that positioning the patient in the prone position after intradural surgery will decrease the CSF pressure at the site of the durotomy, thereby decreasing the flow of CSF through the dural defect ${ }^{2}$. In patients with symptoms such as postural headache, several studies have reported that CLC could be prevented by limiting the patient to the prone position for several days after lumbar duroto$m y^{10,18,20,21)}$. However, there have also been reports that a shortened or even absent bed rest period is not associated with infe- rior outcomes ${ }^{13,19,22)}$. Prolonged bed rest causes multiple complications and increases medical expenses ${ }^{4}$. Therefore, early ambulation could prove beneficial; however, more evidence is required. In the cohort presented here, two of the late group patients had pulmonary thromboembolism and deep vein thrombosis. In addition, the incidence of ileus was significantly higher in the late group. In contrast, early group patients had significantly shorter hospital stays after surgery. Therefore, our findings demonstrate that early ambulation after primary dura closure with Prolene 6-0 sutures could result in reduced health care costs (Fig. 2).

In the present study, the overall incidence of CLC after intradural tumor surgery was $8.39 \%$. While we had expected that the CLC rate of the early group would not be inferior to the late group, the results showed that the late group had a significantly higher CLC rate (early : $2.5 \%$ vs. late : $13.6 \%$, $p=0.006$ ). The most likely reason for these findings was the difference in dura suture material between groups. Based on the experimental evidence, the choice of suture material could result in a disparity in closure strength ${ }^{1,4,9)}$. A previous study using a calf spine model compared braided nylon (Surgilon 5-0; Ethicon Inc.) and Prolene 6-0. Prolene 6-0 was found to produce a more watertight closure at higher hydrostatic pressures $^{4)}$. In the case series presented here, we demonstrated similar outcomes for the clinical utility of Prolene 6-0. Furthermore, the needle diameter for black silk 5-0 was thicker than that for Prolene 6-0 (0.30 vs. $0.25 \mathrm{~mm})$, resulting in a relatively more substantial dura defect along the hole where the needle passed. All dura closures in both groups were performed using a running locked suture technique. We did not investigate the difference between suture techniques, as previous studies have already demonstrated no significant differences in CSF leakage between interrupted and continuous running locked suture techniques ${ }^{4,9)}$.

A widely accepted management option for CLC occurrence is surgical repair either by meticulous direct primary closure of the dura or through augmented closure by means of fat, muscle tissue, or a fascial graft ${ }^{11,21)}$. In our study, CLC was resolved through revision surgery in 19 cases $(6.1 \%, 19 / 314)$. In the early group, only one $(1.3 \%, 1 / 79)$ surgical repair was performed. Guerin et al. ${ }^{11)}$ reported that CSF leaks can be managed by observation alone while on bed rest or by the placement of a shunt either during the index procedure or in the postoperative period. Consistent with this, the late group pa- 
One Day Ambulation after Intradural Surgery | Lee S, et al.

Table 5. Subgroup head to head analysis between laminoplastic laminotomy and laminectomy

\begin{tabular}{lccc} 
& Laminoplastic laminotomy $(\mathbf{n}=190)$ & Laminectomy $(\mathbf{n}=124)$ & $p$-value \\
\hline $\operatorname{CLC}(-)(n=280)$ & $174 / 190(91.6 \%)$ & $106 / 124(85.5 \%)$ & $0.089(0 R, 1.847)$ \\
$C L C(+)(n=34)$ & $16 / 190(8.4 \%)$ & $18 / 124(14.5 \%)$ & $0.089(0 R, 1.847)$ \\
\hline
\end{tabular}

$\mathrm{CLC}$ : cerebrospinal fluid leakage related complications, $\mathrm{OR}$ : odds ratio

tients in our study were routinely ordered to maintain bed rest in the prone position for 3 days or more, and a lumbar drain was inserted during the observation period when pseudomeningocele or high CLC risk was present, based on the judgment of the senior surgeon. Ten patients temporarily maintained CSF shunts with a lumbar drain, and eight of them healed without revision surgery.

In univariate logistic regression analysis for CLC risk factors, inclusion in the late group, revision surgery, and day of postoperative sit-up were all significant. The results showed that the CLC rate increased in proportion to the length of time between surgery and the day of postoperative sit-up. These findings deviated from that which we expected based on causative influences. It is possible that these results are due to the more extended bed rest period experienced by patients in the late group, as well as the postoperative treatment protocol that further prolongs bed rest if empirically assessed as a high risk for CLC. Thus, the postoperative sit-up day factor was excluded in multivariate analysis. Following multivariate logistic regression analysis, inclusion in the late group (primary dura closure using black silk 5-0 suture) and revision surgery (re-exposed through scar) continued to be significant risk factors for CLC. In cases of CSF leakage from the durotomy site, the muscle fascia layer becomes a barrier and prevents CLC. Therefore, a revision surgery patient has delayed wound healing compared to a primary surgery patient. Revision surgery also results in a large dead space due to granular scar tissues, which may have influenced these results. The infection rate might also be influenced for the same reason. In a large scale study of surgical site infections, revision surgery was associated with a significantly higher infection rate than primary surgery ${ }^{24)}$.

The surgical fellow who performed the dural closure ( $p=0.052)$ showed numerical differences in CLC rates, although this was not statistically significant $(0.05<p<0.10)$. The proficiency of the surgical fellows who actually close the dura could affect the CLC rate. Differences in outcomes depending on who performed the dural closure suggest the importance of careful manipulation when performing this procedure. The precise technique required to attain a watertight closing of the muscle fascia while minimizing dead space is also essential.

In our result, laminectomy did not have a statistically significant effect on the CLC rate. CLC occurred in 16 of 190 patients (8.4\%) who underwent laminoplastic laminotomy, and in 18 of 124 patients (14.5\%) with laminectomy by subgroup head to head analysis using the chi-square test $(\mathrm{OR}, 1.847$; $p=0.089$; Table 5). However, laminoplasty after dura closure could reduce CLC rates compared to laminectomy, as demonstrated in a previous meta-analysis comparing the two meth$\mathrm{ods}^{26)}$. A possible explanation for this is that the lamina between the dura and the muscle becomes a barrier and reduces dead space ${ }^{17)}$. Studies in which minimally invasive surgeries were conducted for intradural lesions reported low rates of $\mathrm{CLC}^{8,28)}$. In one such study, the limited soft-tissue exposure and a relative absence of dead space led to a relative increase in epidural pressure, "tamponading" the epidural space and preventing CSF leakage ${ }^{28}$.

In the patient demographics, the mean age was significantly different between the two groups. Advanced age is a risk factor for unintended durotomy that occurs during degenerative spine surger ${ }^{25)}$, and the rate of CLC after incidental dural tear increases with age ${ }^{11}$. However, in another study of the cervical spine, post-operative CSF leak and patient age were irrelevant $^{12}$. Furthermore, it is unclear whether age is a risk factor for CLC after intended durotomy procedures such as intradural tumor surgery ${ }^{2}$. Also, in the present study, patient age was not a significant risk factor by logistic regression analysis.

The present study has several limitations. It was a retrospective study, which prevented randomization of the clinical series of patients. In patient demographics, mean age of both groups were different. Additionally, we investigated two different factors between the groups : suture material and postoperative care strategy with bed rest period. Thus, we were unable to assess whether postoperative 1-day ambulation is 
safe irrespective of suture material. However, the findings described here are meaningful because they demonstrate the efficacy of early ambulation in the context of Prolene 6-0 sutures.

\section{CONCLUSION}

Our findings revealed that intraoperative manipulation had a more significant effect than postoperative management for the prevention of CLC, and that dura suture - needle ratio related to defect along the hole where the needle passed was the most important factor. Dura closure with Prolene 6-0 was associated with significantly lower CLC rates compared to black silk 5-0 with an extended bed rest period, even if early ambulation was begun on the first day after surgery. Postoperative 1-day ambulation combined with Prolene 6-0 sutures appears to be a cost-effective and safe management protocol for intradural spinal surgery.

\section{CONFLICTS OF INTEREST}

No potential conflict of interest relevant to this article was reported.

\section{INFORMED CONSENT}

This type of study does not require informed consent.

\section{AUTHOR CONTRIBUTIONS}

\author{
Conceptualization : SL, JHP \\ Data curation : SCR, KTK \\ Formal analysis : YSL, DCC \\ Funding acquisition : JHP \\ Methodology : DCC, KTK \\ Project administration : SCR, JHP \\ Visualization : DCC, YSL \\ Writing - original draft : SL \\ Writing - review \& editing : SL, SCR, JHP
}

\section{ORCID}

$\begin{array}{ll}\text { Subum Lee } & \text { https://orcid.org/0000-0003-4732-8137 } \\ \text { Dae-Chul Cho } & \text { https://orcid.org/0000-0002-2899-8015 } \\ \text { Kyoung-Tae Kim } & \text { https://orcid.org/0000-0003-4867-6854 } \\ \text { Young-Seok Lee } & \text { https://orcid.org/0000-0002-6881-812X } \\ \text { Seung Chul Rhim } & \text { https://orcid.org/0000-0001-5493-4149 } \\ \text { Jin Hoon Park } & \text { https://orcid.org/0000-0002-0903-3146 }\end{array}$

\section{References}

1. Bakhsheshian J, Strickland BA, Patel NN, Jakoi AM, Minneti M, Zada G, et al. : The use of a novel perfusion-based cadaveric simulation model with cerebrospinal fluid reconstitution comparing dural repair techniques: a pilot study. Spine J 17 : 1335-1341, 2017

2. Barber SM, Fridley JS, Konakondla S, Nakhla J, Oyelese AA, Telfeian AE, et al. : Cerebrospinal fluid leaks after spine tumor resection: avoidance, recognition and management. Ann Transl Med 7 : 217, 2019

3. Bosacco SJ, Gardner MJ, Guille JT : Evaluation and treatment of dural tears in lumbar spine surgery: a review. Clin Orthop Relat Res : 238247, 2001

4. Dafford EE, Anderson PA : Comparison of dural repair techniques. Spine J 15 : 1099-1105, 2015

5. Eismont FJ, Wiesel SW, Rothman RH : Treatment of dural tears associated with spinal surgery. J Bone Joint Surg Am 63 : 1132-1136, 1981

6. Espiritu MT, Rhyne A, Darden BV 2nd : Dural tears in spine surgery. J Am Acad Orthop Surg 18 : 537-545, 2010

7. Fisahn C, Sanders FH, Moisi M, Page J, Oakes PC, Wingerson M, et al. : Descriptive analysis of unplanned readmission and reoperation rates after intradural spinal tumor resection. J Clin Neurosci 38 : 32-36, 2017

8. Formo M, Halvorsen CM, Dahlberg D, Brommeland T, Fredø $\mathrm{H}$, Hald J, et al. : Minimally invasive microsurgical resection of primary, intradural spinal tumors is feasible and safe: a consecutive series of 83 patients. Neurosurgery $82: 365-371,2018$

9. Ghobrial GM, Maulucci CM, Viereck MJ, Beygi S, Chitale A, Prasad S, et al. : Suture choice in lumbar dural closure contributes to variation in leak pressures: experimental model. Clin Spine Surg 30 : 272-275, 2017

10. Grannum S, Patel MS, Attar F, Newey M : Dural tears in primary decompressive lumbar surgery. Is primary repair necessary for a good outcome? Eur Spine J 23 : 904-908, 2014

11. Guerin P, El Fegoun AB, Obeid I, Gille O, Lelong L, Luc S, et al. : Incidental durotomy during spine surgery: incidence, management and complications. A retrospective review. Injury 43 : 397-401, 2012

12. Hannallah D, Lee J, Khan M, Donaldson WF, Kang JD : Cerebrospinal fluid leaks following cervical spine surgery. J Bone Joint Surg Am 90 : 1101-1105, 2008

13. Hodges SD, Humphreys SC, Eck JC, Covington LA : Management of incidental durotomy without mandatory bed rest. A retrospective review of 
20 cases. Spine (Phila Pa 1976) 24 : 2062-2064, 1999

14. Hoover JM, Clarke MJ, Wetjen NM, Mandrekar J, Puffer RC, Krauss WE : Complications necessitating a return to the operating room following intradural spine surgery. World Neurosurg 78 : 344-347, 2012

15. Jenkinson MD, Simpson C, Nicholas RS, Miles J, Findlay GF, Pigott TJ : Outcome predictors and complications in the management of intradural spinal tumours. Eur Spine J 15 : 203-210, 2006

16. Khan MH, Rihn J, Steele G, Davis R, Donaldson WF 3rd, Kang JD, et al. : Postoperative management protocol for incidental dural tears during degenerative lumbar spine surgery: a review of 3,183 consecutive degenerative lumbar cases. Spine (Phila Pa 1976) 15 : 2609-2613. 2006

17. Lee S, Jung SK, Kim HB, Roh SW, Jeon SR, Park JH : Postoperative non-pathological fever following posterior cervical fusion surgery : is laminoplasty a better preventive method than laminectomy? J Korean Neurosurg Soc 63 : 487-494, 2020

18. Lotfinia I, Sayyahmelli S : Incidental durotomy during lumbar spine surgery. Neurosurgery Quarterly 22 : 105-112, 2012

19. Low JC, von Niederhäusern B, Rutherford SA, King AT : Pilot study of perioperative accidental durotomy: does the period of postoperative bed rest reduce the incidence of complication? Br J Neurosurg 27 : 800802, 2013

20. Mayr MT, Subach BR, Comey CH, Rodts GE, Haid RW Jr : Cervical spinal stenosis: outcome after anterior corpectomy, allograft reconstruction, and instrumentation. J Neurosurg 96(1 Suppl) : 10-16, 2002

21. Menon SK, Onyia CU : A short review on a complication of lumbar spine surgery: CSF leak. Clin Neurol Neurosurg 139 : 248-251, 2015

22. Radcliff KE, Sidhu GD, Kepler CK, Gruskay J, Anderson DG, Hilibrand $A$, et al. : Complications of flat bed rest after incidental durotomy. Clin Spine Surg $29: 281-284,2016$

23. Sellin JN, Kolcun JPG, Levi AD : Cerebrospinal fluid leak and symptomatic pseudomeningocele after intradural spine surgery. World Neurosurg 120 : e497-e502, 2018

24. Smith JS, Shaffrey Cl, Sansur CA, Berven SH, Fu KM, Broadstone PA, et al. : Rates of infection after spine surgery based on 108,419 procedures: a report from the Scoliosis Research Society Morbidity and Mortality Committee. Spine (Phila Pa 1976) 36 : 556-563, 2011

25. Smorgick Y, Baker KC, Herkowitz H, Montgomery D, Badve SA, Bachison $C$, et al. : Predisposing factors for dural tear in patients undergoing lumbar spine surgery. J Neurosurg Spine 22 : 483-486, 2015

26. Sun S, Li Y, Wang X, Lu G, She L, Yan Z, et al. : Safety and efficacy of laminoplasty versus laminectomy in the treatment of spinal cord tumors: a systematic review and meta-analysis. World Neurosurg 125 : 136145, 2019

27. Weber C, Piek J, Gunawan D : Health care costs of incidental durotomies and postoperative cerebrospinal fluid leaks after elective spinal surgery. Eur Spine J 24 : 2065-2068, 2015

28. Wong AP, Shih P, Smith TR, Slimack NP, Dahdaleh NS, Aoun SG, et al. : Comparison of symptomatic cerebral spinal fluid leak between patients undergoing minimally invasive versus open lumbar foraminotomy, discectomy, or laminectomy. World Neurosurg 81 : 634-640, 2014 